\title{
Autonomía en disputa: el cuidado en el marco de las relaciones familiares
}

\author{
MARÍA ALICIA GUTIÉRREZ* \\ ANDREA VORIA**
}

\section{Resumen}

La heterosexualidad es un estilo de vida que ha sido hegemónico en los últimos 150 años, regido por la normatividad patriarcal que orienta el deseo hacia la reproducción y organiza el sostenimiento de la vida en términos complementarios (pero no recíprocos) entre los géneros. A través de una norma constrictiva disfrazada de naturaleza, los papeles sociales de la paternidad y la maternidad acaban siendo "biologizados", naturalizados y jerarquizados. La familia se constituye en el espacio de reproducción de relaciones sociales desiguales poniendo en tensión la noción de autonomía, en especial para las mujeres, a pesar de su masiva incorporación a la vida pública. Indagaremos sobre la noción de autonomía frente a las demandas de cuidado que nos son impuestas por vivir en un mundo de seres que, por definición, somos física y psíquicamente vulnerables y dependientes unos de otros, más allá de los dictámenes de la división sexual del trabajo. Proponemos así reconocer la precariedad humana como punto de partida para una redefinición crítica de la noción de autonomía y como práctica de resistencia política.

Palabras clave: heterosexualidad - división sexual del trabajo vulnerabilidad - autonomía - cuidado.

\begin{abstract}
Heterosexuality has been the hegemonic lifestyle for the past 150 years. The patriarchal normativity channels the desire towards reproduction and organizes the sustainability of life in terms of complementarity (but not reciprocity) between genders. A constrictive normative appears as natural, and the roles of paternity and maternity end up appearing as if they were biologically defined, and forming a hierarchy. Family is the center of reproduction of uneven social relations. This places tension upon the concept of autonomy, especially for women, despite their massive incorporation to public life. In this paper we will examine the notion of autonomy in the context of the demands of care that are imposed to us due to the fact that human beings are physically and psychically vulnerable and mutually dependent, even beyond
\end{abstract}

Gutiérrez, María Alicia y Voria, Andrea "Autonomía en disputa: el cuidado en el marco de las relaciones familiares", en Zona Franca. Revista del Centro de Estudios Interdisciplinario sobre Mujeres, Año XXII, No 23, 2014, pp. 11-20.

Recibido: 19 de agosto 2014 - Aceptado: 7 de septiembre 2014 
sexual division of labour. We propose to acknowledge human precariousness as a starting point towards a critical redefinition of the concept of autonomy, and as a practice of political resistance.

Keywords: heterosexuality sexual division of labour vulnerability - autonomy - care

\section{Introducción}

a heterosexualidad, como régimen social, no deja de ser un mito: "un producto histórico y social: el resultado de una época y de unas condiciones sociales determinadas" (Guash, 2000: 17). Es propio de la cultura occidental y no tiene más de 150 años. Como modelo hegemónico, de la mano de la ciencia y la juridicidad, estableció el parámetro de la "normalidad". La especie se sucede por imperativo de la reproducción, que requiere de dos cuerpos significados como varón y mujer: ello instituyó la medicina como la ciencia especializada para reflexionar sobre la sexualidad, o como un cuerpo humano deviene cuerpo sexual (Laqueur, 1994). Entonces la sexualidad también es histórica: "la heterosexualidad es una forma de gestión social del deseo erótico que nace con la revolución industrial, se redefine con la llamada revolución sexual de los años setenta del siglo $X X$ y que ahora está en crisis: en pleno proceso de cambio y transformación" (Guasch y Viñuales, 2003:17).

Dicha "naturalidad" es puesta en cuestión por las ciencias sociales, la teoría feminista, la antropología y la filosofía, entre otras. Surgen así los estudios sobre sexo, sexualidad, género que organizan el sentido de la sociedad, en reemplazo de la religión que suturaba el orden social y de la vida.

El quiebre de este orden en la revolución de las luces, permite el surgimiento del individuo como ente supremo, por sobre el sentido de la comunidad. Este sujeto universal, autónomo, se instituye en los límites de la corporalidad. Sujeto escindido en el principio cartesiano: razón y emoción que instaura como suprema la razón dentro del imperativo del contrato que funda las llamadas sociedades modernas. Dicho sujeto produce un entramado o una maraña con otros/ as en una matriz (Rich, 1986) en un régimen político heterosexual (Wittig, 1992) que establece regulaciones, órdenes y funciones del género. La conformación de la heterosexualidad como norma, la estructuración del sistema capitalista/patriarcal, la división sexual del trabajo, la apelación a un sujeto autónomo en una trama de dependencia y vulnerabilidad, pondrán en evidencia la importancia de reformular la histórica "ética del cuidado" en función de una lógica que considere la autonomía dentro de un proceso social en construcción.

\section{El sexo y las diferencias de género}

Las teorías del sistema sexogénero evidenciaron la organización estructural de la sociedad. Gayle Rubin (1986) articula la noción de sistema sexo/género a partir de la idea que la condición de macho o hembra comporta necesidades en común: comer, dormir y defecar. La vulnerabilidad del sujeto al nacer, por su "incompletud" y que se reedita en la ancianidad, le permite plantear que la "diferencia" no existe en la condición de la naturaleza. La construcción social privilegia aquellos aspectos de la diferencia corporal que se necesitan para la reproducción humana. La autora acuña el concepto de sistema sexo/género para referirse a los modos en que la materialidad del sexo es convertida por las relaciones sociales desiguales en un sistema de prohibiciones, obligaciones y derechos diferenciales para hombres y mujeres. Esta díada se sustenta en las diferencias "naturales" que el sexo podría demarcar (por oposición al género construido culturalmente) pero sin cuestionar el binarismo sexual (Pierucci, 1999). De acuerdo a Torrás (2007), existe una normativización jerarquizada que hace legibles los cuerpos tras una supuesta construcción biológica.

Esa jerarquización ubicó a hombres y mujeres (definidos por supuestos atributos naturales) en posiciones diferenciales: varones-proveedores en el ámbito público, mujeres-cuidadoras en el mundo privado. El ámbito privado reservado a las mujeres se sustenta en el mito de la maternidad, función supuestamente exclusiva de sujetos clasificados como mujeres a quienes se les confiere la función y la responsabilidad de la reproducción y del cuidado de las personas dependientes. En este esquema la heterosexualidad y la reproducción son estatutos naturales e incuestionables.

El feminismo de la segunda ola redefine esta división sexual, pero sin correrse del sistema binario: no somos solo úteros. La maternidad, ligada por el orden de la naturaleza a las mujeres, no implica necesariamente la función social; responde a la condición de subordinación de las mismas. Esta lógica se inscribe en la estructura patriarcal y en el régimen político heterosexual y se expresa a través de la violencia, el poder, la desigualdad que producen la 
domesticación de los cuerpos (Segato, 2003). Distintas autoras entienden el patriarcado de manera diversa lo que muestra las tensiones que se producen en la relación género, sexualidad y clase social.

Millet entiende al patriarcado como una estructura de opresión y dominación que despliega el poder masculino sobre las mujeres en todos los contextos de la vida, tanto públicos como privados, aunque es en la familia donde tiene su origen y donde ejerce su fuerza mayor, a través de la asignación de roles de género (Millet, 2010). La dependencia económica de las mujeres refuerza sus dificultades para la autonomía y la autodeterminación. Las feministas radicales ponen en tensión el sentido del patriarcado al plantear que la revolución feminista radical solo podrá ser realizada con el desarrollo de las técnicas de reproducción artificial dado que la raíz de la opresión de la mujer está en su servidumbre biológica, en la maternidad (Firestone, 1976). La familia biológica constituye una distribución del poder desigual. Por la diferenciación reproductiva del orden de la naturaleza se deslizan las diferencias en la división sexual del trabajo. Por ello reconoce que es un problema esencialmente político, que refiere a las relaciones de poder. Se introduce, entonces, la idea de doble opresión con las nociones de producción y reproducción (Mitchell, 1974) que indica la existencia de una relación dialéctica entre la estructura de clases capitalista y la estructuración sexual jerarquizada (Eisenstein, 1978)

Heidi Hartman en su clásico texto El infeliz matrimonio entre marxismo y feminismo: hacia una unión más progresista define al patriarcado como "un con- junto de relaciones sociales que tiene una base material y en la cual hay relaciones jerárquicas entre los hombres y solidaridad entre ellos, lo que les permite dominar a las mujeres. La base material del patriarcado es el control de los hombres sobre las mujeres, en la esfera de la producción, negando el acceso a las mujeres a los recursos productivos económicamente necesarios y restringiendo su sexualidad" (Hartman, 1983: 11). Esto lleva a la relación de mutua legitimación/deslegitimación entre el capital y el patriarcado (el lugar de la familia, la autonomía de las mujeres, la competencia en el mercado laboral). Propone "una sociedad en la cual el reconocimiento de la interdependencia es liberación antes que vergüenza, el cuidado y la crianza de los niños es una práctica universal, no opresiva, y en la cual las mujeres no deben continuar siendo el soporte de las libertades, tanto falsas como concretas, de los hombres" (Hartman, 1983: 17).

La crítica más sustantiva a la posición de Hartman la desarrolló Iris Young (1992) con su artículo Marxismo y Feminismo: más allá del "matrimonio infeliz" (una crítica al sistema dual), quien postula que el proyecto del feminismo socialista "debe ser el desarrollar una teoría única, aprovechando lo mejor del marxismo y del feminismo radical, para comprender al patriarcado capitalista como un sistema en el cual la opresión de la mujer sea un atributo central" (Young, 1992: 1). Algunas teóricas del sistema dual plantean al patriarcado como un modo de producción en sí mismo, que coexiste al lado del modo de producción capitalista. Otras autoras van a referirse a dos sistemas estructurados como "modo de producción" y "modo de reproducción". El modelo de esferas separadas "al asumir que la esfera primaria de las relaciones patriarcales es la familia, no repara en el carácter y el grado de opresión específico de las mujeres, en tanto tales, fuera de la familia. (Young 1992:4). En lo más mundano -dirá Younguna teoría del sistema dual no parece tener las herramientas teóricas para identificar y analizar las formas específicas de opresión sexista que las mujeres sufren actualmente en sus puestos de trabajo. Expresará que es necesario crear una teoría de las relaciones de producción y de las relaciones sociales de ellas derivadas que "conciban a las relaciones de género y a la situación de las mujeres como elementos centrales" (Young 1992:12).

Es evidente que el patriarcado, en todas las versiones de las teorías feministas, se constituye sobre la base de la violencia sexual, el control de los cuerpos en general y el de las mujeres en particular. El control de los cuerpos, según Silvia Fedirici (2011) fue el elemento "estabilizador" del proceso de acumulación originaria del capital. Dicho control implica tener acceso al proceso de reproducción, piedra angular de la reproducción de la fuerza de trabajo. La mujer/naturaleza tiene la función esencial de equilibrar el crecimiento demográfico y la "calidad" de la reproducción.

Así entendemos que el patriarcado está sostenido sobre una doble dimensión: por un lado, la dimensión socio-económica que garantiza el sostenimiento de la vida en términos materiales; y, por el otro, la dimensión psíquica-emocional que se constituye en el mecanismo por excelencia de orientación del deseo, en respuesta a las exigencias estructurales.

Sin embargo, dichas posicio- 
nes no tienen realidad más allá de su sostenimiento a través de la performatividad (Butler, 1990) de los sujetos, en el marco de una red de relaciones necesarias. Que dichas relaciones se sustenten a través de su práctica cotidiana, nos lleva a interrogarnos sobre aquello que las vuelve tan precarias y a la vez tan consistentes, así como sobre el cuidado como eje central que vertebra las condiciones de desigualdad y las tensiones de las lógicas de género.

\section{El "cuidado" en tanto categoría}

Abordar el análisis de la categoría "cuidado" supone pensar el modo en que hombres y mujeres sostenemos la vida en el marco de sociedades patriarcales capitalistas, tanto en lo que se refiere a la producción económica como a las tareas de cuidado. Sin embargo, ha sido una categoría tradicionalmente descuidada (por no decir, invisibilizada, naturalizada, ocultada), en consonancia "con una visión de la familia como un solo grupo de interés y como agente de cambio" (Hartmann, 2000: 17).

Por el contrario, la categoría "cuidado" -por lo menos entendida en los términos que proponemos a continuación- es una dimensión de análisis que nos lleva a pensar a la familia como lugar de conflicto y de disputa de intereses. Así, tanto los estudios de género, el pensamiento feminista, como el movimiento de mujeres han colaborado -tanto desde la acción política como desde la producción académica- en desnaturalizar el "cuidado" como un don, como un "regalo" de las mujeres hacia las personas dependientes (niños/ as, enfermos/as, ancianos/as o discapacitados/as), otorgándole la categoría de "trabajo" y cues- tionando la transferencia unilateral a nivel social de dicho rol a las mujeres en tanto esposas, madres y amas de casa.

La incorporación masiva de las mujeres a la vida pública de nuestra sociedad -a nivel educativo, político como del mercado de trabajo- le ha impreso tal urgencia al tema, que el sostenimiento de la vida a nivel de las demandas de cuidado de nuestros hogares se ha vuelto un terreno de disputa tanto a nivel de las relaciones personales, como en términos de demandas de derechos y ciudadanía. Tal es la envergadura de la llamada crisis del cuidado como síntoma de emancipación de las mujeres (Montaño, 2010) que autoras como Aguirre y Batthyány (2007) consideran que esta problemática se encuentra en el centro de "la nueva cuestión social".

Así, obtener ingresos ha dejado de ser una responsabilidad exclusiva de los hombres. Lo demuestra la reciente historia argentina en el marco de la crisis de 2001, donde ante la urgencia de garantizar la supervivencia del grupo familiar, las mujeres se incorporan masivamente al mundo del trabajo. Si bien su incorporación se dio en gran medida en condiciones informales y en un mercado de trabajo segregado en términos de género, dicha participación generó movimientos tanto a nivel subjetivo como colectivo que les permitió cuestionar si la atención a las personas era una responsabilidad exclusiva de ellas.

De modo que, frente a las férreas fronteras de la división moderna entre lo público y lo privado -que sostienen estructuralmente la división y fragmentación de la división sexual del trabajo-, nos proponemos pensar más bien la porosidad de dichos contornos, en tanto no hay garantías para sostener el cuidado de la vida sin una doble estructura tanto pública como privada, que lo habilite y lo sustente.

\section{Sosteniendo la vida}

Consideramos relevante problematizar acerca del modo en que hombres y mujeres sostenemos la vida en un sistema patriarcal capitalista, el cual nos posiciona en una estructura de relaciones dependientes (pero no recíprocas), según una lógica de complementariedad que se conforma, como ya vimos, bajo la dimensión socioeconómica y la psíquica emocional. Esta doble estructuración del patriarcado hace que los procesos de transformación de las relaciones patriarcales entrañen dificultades añadidas, ya que cuestionan la raíz misma de la identidad de las personas, y no sólo el lugar que ocupan en el mundo (Izquierdo, 1998). De este modo, son estructuras que suelen reforzarse, una a la otra, con el objetivo de sostener la estabilidad del sistema, no sólo a nivel de posiciones sociales, sino de la conformación de deseos y aspiraciones (Voria, 2007).

La posición social hombre es viable por la existencia de la posición mujer, y ambas posiciones quedan definidas por la división sexual del trabajo. De modo equivalente, la posición psíquica masculina es viable y se sostiene por la existencia de la posición psíquica femenina (Izquierdo, 2009). Sin embargo, dichas posiciones no tienen realidad más allá de su sostenimiento a través de la performatividad de los sujetos (Butler, 1997, 1998), en el marco de una red de relaciones necesarias pero incompleta, en tanto no se fija en un conjunto estable de diferencias discursivas (Laclau 
y Mouffe, 2004). De modo que, al hablar de identidades de género, consideramos que toda identidad es relacional y que dichas relaciones tienen un carácter necesario, que se deriva de la regularidad de un sistema de posiciones estructurales de género (Voria, 2012), que toma la ordenación de la sexualidad como fundamento del orden social.

La performatividad de género supone que el sujeto es alguien que no puede ser sin hacer (Butler, 1997, 1998), de modo que cualquier fracaso en la formación de sujetos genéricos es un efecto de tener que formarse en el tiempo una y otra vez, abriendo un resquicio a posibles fallos o fisuras (Voria, 2011).

Es en la confluencia del sistema patriarcal y capitalista donde podemos pensar a nivel estructural el sostenimiento de nuestra vida material, a partir tanto de la división sexual del trabajo en el seno de la familia patriarcal, como de la división social del trabajo entre los propietarios de los medios de producción y los/ as trabajadores/as. Que dichas relaciones se sustenten a través de nuestra práctica cotidiana, es lo que las vuelve tan precarias y a la vez tan consistentes (Izquierdo, 1998).

Es la opresión de las mujeres en la modernidad la que muestra el nexo entre patriarcado y capitalismo. Las mujeres, a través de su trabajo doméstico, se convierten en piezas clave de la obtención de ganancias por parte de los capitalistas, en la medida que son ellas las encargadas de brindar los cuidados necesarios para el sostenimiento de la mano de obra trabajadora. Como señala Young, a través de este vínculo de explotación se transfieren los resultados del trabajo de las mujeres en beneficio de los hombres. Estas relaciones se producen y reproducen a través de un proceso sistemático, pero no recíproco, en el cual las mujeres se dedican a mantener y aumentar el poder, categoría y riqueza de los hombres (Young, 2000).

Analizar la dimensión material del patriarcado supone, entonces, pensar desde una perspectiva de género no sólo el modo en que las personas producen sus vidas, sino también el tipo de relaciones sociales que establecen para producirlas; es decir, en qué consiste el trabajo, quién hace qué y para quién, cómo se recompensa el trabajo y cuál es el proceso social por el cual las personas se apropian de los resultados del trabajo, dado que esto nos permite vislumbrar las relaciones de poder y desigualdad entre los géneros, que desembocan en relaciones estructurales de explotación.

Por tanto, una lectura crítica a la noción de "cuidado" supone considerar las relaciones de explotación no sólo sobre las que se da respuesta a las necesidades vitales de las personas dependientes, sino también sobre las que se sostiene el sistema patriarcal capitalista imperante, que se niega en reconocer la vulnerabilidad constitutiva de la especie humana. En este sentido, para Young, "hacer justicia donde hay explotación requiere reorganizar las instituciones y las prácticas de toma de decisiones, modificar la división del trabajo, y tomar medidas similares para el cambio institucional, estructural y cultural" (Young, 2000: 93).

\section{El cuidado y sus ambivalencias}

La división sexual del trabajo es una característica de la organización de las actividades productivas, y es también un mecanismo de socialización que tiene efecto en la construcción de las subjetividades de hombres y mujeres, con sesgo de género.

De este modo, entendemos el proceso de producción del ser humano como la producción física de su vida, de la producción de los medios que la hacen posible y, más aún, de la producción de sentido de su vida. Esta última definición del proceso de producción de hombres y mujeres nos permite pensar y analizar el trabajo como una dimensión más compleja de la vida humana, que toma en cuenta no sólo el sostenimiento material de nuestra especie, sino también aquello que le da sentido a nuestro tiempo, a nuestro esfuerzo.

De acuerdo con la división sexual del trabajo, las actividades productivas de los hombres se orientan a procurar sus medios de vida y los de su familia. En consecuencia, la manera como expresan el amor y la responsabilidad que experimentan hacia las personas que dependen de ellos es -en términos normativos- mediante los recursos que pueden aportar al hogar (lo cual no significa que los sujetos concretos no guarden distancia performativamente en relación a lo que indica la norma). Al mismo tiempo, se suelen encontrar desencajados ante la demanda de atención personal de su familia, sin los recursos emocionales y las destrezas necesarias interiorizadas muchas veces para dar respuesta a los requerimientos que requiere el cuidado.

En cambio, las actividades designadas normativamente como femeninas van encaminadas a la satisfacción directa de las necesidades humanas, los bienes o servicios que producen tienen un valor que se manifies- 
ta en el uso y se mide por la satisfacción que proporcionan a otro directo. De este modo, la producción de la mujer adquiere su valor de un modo contextual o concreto, al momento en que las personas encuentran satisfacción en las tareas de cuidado recibidas, con lo cual no se establece un patrón de valorización universal, sino que depende de las circunstancias, el tipo de relación, las emociones que predominan en el vínculo, etc. (Izquierdo, 2003).

Por tanto, así como nos podemos referir a una ética específica asociada al cuidado, también podemos hablar de una ética de la provisión-protección. Esta última es la que explicaría el que los hombres sean explotables y se sometan a condiciones de trabajo degradantes y empobrecedoras, poniendo en riesgo su vida, implicándose en acciones dañinas para la salud y, en último extremo, participando activamente en conflictos bélicos ${ }^{1}$.

La ética del cuidado, tal como ha sido entendida por la modernidad, supone una situación de dependencia unidireccional, que ubica en posiciones fijas a la figura de cuidadora y a la persona objeto de sus cuidados. No se concibe que todos/as somos a la vez cuidadores/as y objetos de cuidado. Pero no sólo se trata de cómo las mujeres hacen viable la vida humana a través de cierta cooperación social en relación a las demandas de cuidado propias de la especie humana-, sino también el modo en que queda expuesta su subjetividad a los mandatos estructurales que direccionan sus deseos a satisfacer las necesidades ajenas. Como consecuencia, a pesar que las mujeres nos hayamos incorporado masivamente al mercado de trabajo, como es el caso en Argentina, no hemos encontrado todavía el camino para elaborar estrategias que permitan superar el sentimiento de culpabilidad que acompaña el corrimiento respecto al mandato tradicional del cuidado (Federici, 2013).

En este escenario hostil que suele privatizar e intimizar nuestras experiencias, Carolina del Olmo $(2013,2014)$ se pregunta sobre los modos de sostener la maternidad, lo cual le permite reflexionar sobre los sentidos del cuidado. Según ella, ni las teorías del apego (niñocéntricas) ni los mandatos paternos (adultocéntricos) resuelven la tensión en las sociedades modernas. La recuperación de la "tribu" como espacio social del cuidado, permitiría preservar según ella la autonomía en un sentido de confluencia y solidaridad y a su vez asumir la responsabilidad colectiva del cuidado.

Por tanto, lo interesante respecto a este tema es hacer una revisión de esta normatividad hegemónica -todavía hoy en nuestra sociedad, a pesar de su continua metamorfosis- en dos sentidos: por un lado, otorgarles más allá de la dimensión ética, un carácter político, en tanto coarta el recorrido vital de los sujetos femeninos y masculinos, generando costes y beneficios diferenciales (y desiguales) para cada género. Por el otro lado, su contenido ético exige ser revisado en tanto responsabilidad recíproca, y no en términos del carácter subsidiario que se le adjudica a los hombres en el cuidado de las personas dependientes, y a las mujeres respecto a su participación en el mercado de trabajo.

\section{Los límites de la noción de soberanía}

La cuestión del cuidado coloca en una encerrona a la noción moderna de autonomía en tanto pone en el centro la vulnerabilidad y la dependencia mutua como constitutiva del ser humano, que el sistema imperante ha negado históricamente sobre la base de la ficción de la autosuficiencia del individuo liberal moderno. Aún más, la autonomía ha sido y es actualmente una de las demandas que vertebran históricamente al movimiento feminista y de mujeres en relación a las luchas por el derecho al aborto legal, a los derechos sexuales y reproductivos y en contra la violencia de género. Sin embargo, hay una discusión a plantear, incluso dentro del seno del propio feminismo. Izquierdo lo expresa contundentemente al referir que "La idea del selfmade man, de ese hombre que no le debe nada a nadie puesto que se ha hecho a sí mismo es una fantasía omnipotente que forma parte de la mitología de las democracias modernas [...] es un indicio de un modo peculiar de socialización que lleva a las personas a no ser capaces de reconocer el peso de las circunstancias y de la historia, a censurar una parte de su biografía" (Izquierdo, 2003: 5-6).

Como bien sabemos a esta altura, la noción liberal de autonomía presupone un sujeto que está en absolutas condiciones de racionalidad para sus decisiones, negando que está instituido como tal en un no saber del inconsciente que condiciona muchas de sus decisiones y en una articulación de ese inconsciente como un lenguaje complejo de descifrar. De modo que, el individuo autónomo en la toma de decisiones para su vida está condicionado por su conformación en un contexto epocal y cultural determinado.

Por tanto, el dilema del cuidado difícilmente encuentre respuesta desde la noción liberal de autonomía, en tanto es una visión que no deja resquicio a 
la vulnerabilidad humana y a la interdependencia. Se trata, en cambio, de abordar dicho concepto en términos críticos y transformadores de las relaciones sociales, dando el salto entre la dimensión moral individual de la autonomía a su dimensión política colectiva (Gutiérrez, 2010).

El desafío consiste en repensar la categoría "cuidado" en términos de la sostenibilidad de la vida humana de un modo relacional, en su voz activa y en su voz pasiva. Se trata tanto de cuidar (incluso cuidarse a sí misma/o), como de ser cuidada/o, en distintos grados, dimensiones y formas a lo largo del ciclo vital. Cuestionamos, así, el par autonomía / dependencia sobre el que tradicionalmente se ha sostenido el concepto de cuidado, para reclamar por el de interdependencia social: "las personas no somos autónomas o dependientes, sino que nos situamos en diversas posiciones en un continuo de interdependencia" (Pérez Orozco, 2006: 14).

Si hacemos una lectura tanto de los logros alcanzados como de las transformaciones todavía hoy pendientes, quizá la lucha por la autonomía de las mujeres debe ser reorientada hacia una lucha que posicione el problema del cuidado como responsabilidad de todos/as y de cada uno/a, como lo es también el de la producción de bienes, partiendo de la idea butleriana (2006) que la vulnerabilidad atraviesa y configura no sólo nuestro ser vital como también político. Así, la autonomía se delinea como proyecto (Castoriadis, 1990), en su doble dimensión personal y colectiva. Esto nos lleva a repensar, incluso, si se trata de un estado o más bien de "un movimiento sin fin" (Castoriadis, 1990: 84), entendiendo la autonomización como un proceso incesante de construcción y deconstrucción junto al otro, abriendo un espacio de interrogación sin límites tanto en el orden de lo individual como de lo social.

Para finalizar, nos interesa rescatar aquí el modo en que Butler enlaza en sus últimos escritos $(2012,2014)$ la cuestión de la vulnerabilidad humana, en relación a su noción de agencia alejada, a su entender, de modos de acción soberanos (liberales y patriarcales). La agencia para Butler (1997) comienza allí donde la soberanía termina, considerando la performatividad como una intervención comprometida en un proceso interminable de repetición y citación.

Butler reconoce sobre los riesgos que supone colocar la vulnerabilidad humana en el centro y lejos está su intención de naufragar en las mismas orillas, según ella, de la ética del cuidado o el pensamiento maternal, que apuntale el poder patriarcal. Por el contrario, su propuesta es más radical: se trata de movilizar y poner en escena la vulnerabilidad humana como práctica misma de resistencia política (Butler, 2014). De modo que el dilema del cuidado debería adquirir la suficiente fuerza y resistencia en términos de demanda de ciudadanía, no sólo para problematizar sobre la precariedad que nos constituye como especie, sino para visibilizar aquella red de infraestructura, servicios y derechos postergados, que atentan contra el sostenimiento mismo de la vida en términos sociales.

\section{Comentario final}

A través de las consideraciones desarrolladas en el presente artículo pretendimos mostrar cómo se constituye, en términos de poder, el régimen heterosexual que se sustenta en normas performativas que diseñan un modo de la vida centrado en la diferencia sexual. Esa diferencia expresada en la organización patriarcal de la división sexual del trabajo, se materializa, entre otras expresiones, a través de las lógicas del cuidado que hacen al sostenimiento de la vida y a la aceptación de la condición de vulnerabilidad que nos alberga en tanto humanos. Dicha interdependencia pone en cuestionamiento la noción liberal de autonomía y la agencia si no es inscripta en una sociedad soberana.

En este escenario, se actualiza la pregunta sobre la autonomía de las mujeres que formula Laura Pautassi, “¿Qué autonomía se puede reclamar en la medida que existan personas que hay que cuidar?"(2007: 36). Dicha pregunta difícilmente encuentre respuesta desde la noción liberal de autonomía, en tanto es una visión que no deja resquicio a la vulnerabilidad humana y a la interdependencia.

En este sentido, Del Olmo se plantea "valoramos la independencia y la autonomía más allá de lo razonable y nos empeñamos en no ver que para llegar a ser -o fingir que somosautónomos todos hemos pasado y pasaremos de nuevo por etapas de radical dependencia, en las que sólo una densa red de relaciones personales nos ha permitido sobrevivir y madurar. Una red de la que, en el fondo, seguimos dependiendo en todo momento" (Del Olmo, 2014).

En definitiva, el dilema al que nos enfrentamos radica en cómo incorporar a las luchas por la autonomía las demandas de cuidado que nos son impuestas por vivir en un mundo de seres que, por definición, somos física y psíquicamente vulnerables 
y dependientes unos de otros, más allá de los dictámenes de la división sexual del trabajo.

Reconociendo la precariedad humana como punto de partida para una redefinición crítica de la noción de autonomía, proponemos aquí orientarnos en futuros recorridos hacia un concepto de autonomía sensible al género, encarnado sobre la base material de la vulnerabilidad de nues- tros cuerpos y de la dependencia mutua que ello supone en términos psíquicos como sociales -no ya en términos unilaterales sino recíprocos-, donde el cuidar o ser cuidada/o no sean posiciones estancas y desiguales, sino roles intercambiables en relación a las circunstancias vitales e históricas vividas.

De lo que se trata, en última instancia, para Carrasco es de asumir como única utopía posible "apostar a fondo por el sostenimiento de la vida humana" (2001: s/n). Así, la clave radica en que hombres y mujeres nos comprometamos en sostener la vida de un modo recíproco, fuera de los cánones patriarcales, asumiendo nuestra mutua dependencia en relación a un otro, que nos permita afirmar y reconocer nuestra humanidad.

Notas:

* Socióloga, Profesora Titular de la Universidad de Buenos Aires, Argentina. Directora de Proyecto UBACYT Grupos Consolidados (2013-2016).mariagut27@ yahoo.com.ar

** Magíster y Doctora en Sociología por la Universidad Autónoma de Barcelona. Profesora titular de la Universidad del Salvador. Investigadora externa UBACYT Grupos Consolidados (2013-2016) andreavoria@gmail.com

1. Aclaremos que nos desplazamos en un terreno analítico, y no descriptivo de las subjetividades individuales, donde pueden convivir rasgos propios de la feminidad y la masculinidad. Ahora bien, si podemos hablar de sexismo "es porque tendencialmente, en las mujeres predomina un cierto tipo de rasgos y se espera de ellas que los tengan, mientras que en los hombres son otros los rasgos que prevalecen y se espera de ellos que les caractericen" (Izquierdo, 2003: 3).

\section{Bibliografía:}

AGUIRRE, R. y BATTHYÁNY, K. (2007) "Introducción", en Gutiérrez, María Alicia (comp.), Género, familias y trabajo: rupturas y continuidades. Desafíos para la investigación política. Buenos Aires: Consejo Latinoamericano de Ciencias Sociales - CLACSO.

BUTLER, Judith (1990) "Variaciones sobre sexo y género. Beauvoir, Wittig y Foucault" en Benhabib, Seyla y Cornella Drucilla, Teoría feminista y teoría crítica. Valencia: Edicions Alfons el Magnànim.

BUTLER, Judith (1997) Lenguaje, poder e identidad. Editorial Síntesis, Madrid.

BUTLER, Judith (1998) "Actos performativos y constitución del género: un ensayo sobre fenomenología y teoría feminista", en Debate Feminista, vol. 18, octubre, México, pp. 296-314

BUTLER, Judith (2002) "Críticamente subversiva”, en Mérida Jiménez, Rafael (ed.), Sexualidades transgresoras. Una antología de estudios queer. Barcelona: Icaria.

BUTLER, Judith (2006) Deshacer el género. Barcelona: Paidós Ibérica.

BUTLER, Judith (2012) "Cuerpos en alianza y la política de la calle", en Revista Transversales, número 26, junio, Buenos Aires.

BUTLER, Judith (2014) "Repensar la vulnerabilidad y la resistencia", en XV Simposio de la Asociación Internacional de Filósofas. Alcalá de Henares, 24 de junio.

BUTLER, Judith y LACLAU, Ernesto (1999). "Los usos de la igualdad", en Debate Feminista, Nro. 19, México, pp. 115-139.

CARRASCO, Cristina (2001) "La sostenibilidad de la vida humana: ¿un asunto de mujeres?", en Revista Mientras tanto, Nro. 82, otoño invierno, Barcelona, pp. 43-70. 
CASTORIADIS, Cornelius (1990) La institución imaginaria de la sociedad. Vol.2 El imaginario social y la institución. Tusquets Editora, Buenos Aires.

DEL OLMO, Carolina (2013) ¿Dónde está mi tribu? Maternidad y crianza en una sociedad individualista. Capital Intelectual, Buenos Aires.

DEL OLMO, Carolina (2014) "Sufrir y gozar, la maternidad ambigua", en Revista N, 22 de septiembre.

EISENSTEIN, Zillah (1978) Capitalist Patriarchy and the Case for Socialist Feminism, Monthly Review Press, NYC.

FEDERICI, Silvia (2013) Revolución en punto cero. Trabajo doméstico, reproducción y luchas feministas. Traficantes de sueños, Madrid.

FEDERICI, Silvia (2011) Calibán y la Bruja. Mujeres, cuerpo y acumulación originaria, Editorial Tinta Limón, Buenos Aires.

FIRESTONE, Shulamith (1976) La dialéctica del sexo. En defensa de la revolución feminista, Editorial Kairos, Barcelona.

GUASCH, Oscar y VIÑUALES, Olga (Eds.) (2003) Sexualidades. Diversidad y Control Social, Ediciones Bellaterra, Barcelona.

GUASCH, Oscar (2000) La crisis de la heterosexualidad, Editorial Laertes, Barcelona.

GUTIERREZ, María Alicia (2010) "Autonomía y Libertad: acerca del aborto y su implicancia en el cuerpo de las mujeres" en Anais Eletrónicos, Fazendo Género 9, Universidad de Santa Catarina, Florianópolis, Brasil, septiembre, CD, ISSN 2179-510X.

HARTMANN, Heidi (1983) "El infeliz matrimonio entre marxismo y feminismo: hacia una unión más progresista", en Teoría y Práctica, México, pp. 12-13.

HARTMANN, Heidi I. (2000) "La familia como lugar de lucha política, de género y de clase: el ejemplo del trabajo doméstico", en Navarro, Marysa y Stimpson, Catharine (comp.), Cambios sociales, económicos y culturales. Buenos Aires: Fondo de Cultura Económica, pp. 17-58.

IZQUIERDO, María Jesús (1998) El malestar en la desigualdad. Ediciones Cátedra, Madrid.

IZQUIERDO, María Jesús (2001) "Razón y sentimiento en las relaciones de pareja: ¿Del contrato al diálogo?". Ponencia presentada en el Congreso Los hombres ante el nuevo orden social. Donostia: Emakunde, España, 13 al 15 de junio.

IZQUIERDO, María Jesús (2003). "Del sexismo y la mercantilización del cuidado a su socialización: Hacia una política democrática del cuidado". Ponencia presentada en el Congreso Cuidar cuesta: costes y beneficios del cuidado. Donostia: Emakunde, España, 13-14 de octubre.

LACLAU, Ernesto y MOUFFE, Chantal (2004). Hegemonía y estrategia socialista. Hacia una radicalización de la democracia. Fondo de Cultura Económica, Buenos Aires.

LAQUEUR, Thomas (1994) La construcción del sexo. Cuerpo y género desde los griegos hasta Freud, Ediciones Cátedra, Madrid.

MILLET, Kate (2010) Política Sexual, Colección Feminismos, Ediciones Cátedra, Universidad de Valencia, Valencia.

MITCHELL, Juliet (1974). Psychoanalysis and feminism. Vintage Books, New York.

MONTAÑO VIRREIRA, Sonia y CALDERÓN MAGAÑA, Corral (coord.) (2010) "EI cuidado en acción. Entre el derecho y el trabajo", Cuadernos de la CEPAL 94, Santiago de Chile: CEPAL, AECID, UNIFEM.

PAUTASSI, Laura (2007) El cuidado como cuestión social desde un enfoque de derechos. Serie Mujer y Desarrollo № 87, Santiago de Chile: CEPAL.

PÉREZ OROZCO, Amaia (2006). "Amenaza tormenta: la crisis de los cuidados y la reorganización del sistema económico", en Revista de Economía Crítica, n 5 , marzo, Valladolid, pp. 7-37.

PIERUCCI, Antonio Flávio. (1999) Ciladas da Diferentia. Editora 34, San Pablo.

$\mathrm{RICH}$, Adrienne (1986) Heterosexualidad obligatoria y existencia lesbiana, en Revista Brujas (Asociación de Trabajo y Estudio de la Mujer-ATEM), año 4, Nos. 10, 11, 12, Buenos Aires.

RUBIN, Gayle (1986) "El tráfico de mujeres: notas sobre la economía política del sexo" en Revista Nueva Antropología, noviembre, Volumen VIII, № 30, México, pp.95-145.

SEGATO, Rita (2003) Las estructuras elementales de la violencia. Ensayos sobre género entre la antropología, el psicoanálisis y los derechos humanos, Editorial Prometeo/Universidad Nacional de Quilmes, Buenos Aires Argentina. 
TORRAS, Meri (2007) "El delito del cuerpo. De la evidencia del cuerpo al cuerpo en evidencia" en Meri Torras (ed.). Cuerpo e identidad I. Barcelona: Edicions UAB.

VORIA, M. Andrea (2012) "Género, performatividad y discurso: Obediencias y transgresiones en pugna" en Revista Encuentros Latinoamericanos Sección Estudios de Género. Montevideo.

VORIA, M. Andrea (2011) "Géneros contingentes. Luchas por el reconocimiento en contextos de crisis social”. En Gutiérrez, María Alicia (comp.), Voces polifónicas: Itinerarios del género y las sexualidades. Buenos Aires: Editorial Godot, Colección Crítica, pp. 31-65.

VORIA, M. Andrea (2007) Conversaciones en la ciudad desde una mirada de género, Alcalá de Henares: Ayuntamiento de Alcalá de Henares. Premio de Investigación María Isidra de Guzmán XV Edición.

WITTIG, Monique (1992) El pensamiento heterosexual y otros ensayos, Editorial Egales, Barcelona y Madrid.

YOUNG, Iris Marion. (1990) "Imparcialidad y lo cívico público. Algunas implicaciones de las críticas feministas a la teoría moral y política", en Seyla Benhabib y Drucilla Cornella (Eds.), Teoría feminista y teoría crítica. Valencia: Edicions Alfons el Magnànim.

YOUNG, Iris Marion (1992) "Marxismo y feminismo: más allá del matrimonio infeliz (una crítica al sistema dual)" en El Cielo por Asalto, Año II, N 4, Ot/ Inv., Argentina.

YOUNG, Iris M. (2000) La justicia y la política de la diferencia. Madrid: Ediciones Cátedra. 